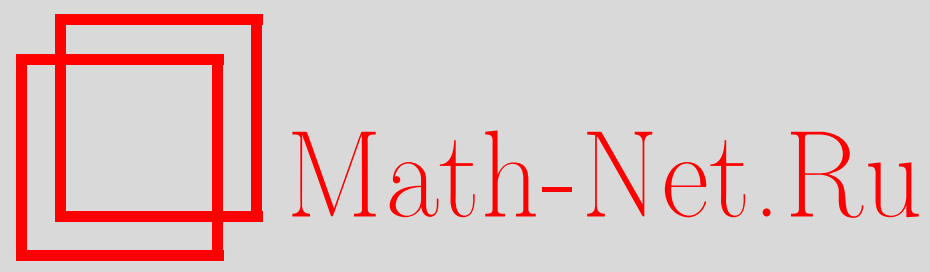

В. А. Смирнов, Гомотопические теории алгебр над операдами, Матем. заметки, 2005, том 78, выпуск 2, 278-285

DOI: https://doi.org/10.4213/mzm2650

Использование Общероссийского математического портала Math-Net.Ru подразумевает, что вы прочитали и согласны с пользовательским соглашением http://www.mathnet.ru/rus/agreement

Параметры загрузки:

IP : 54.209 .52 .79

26 апреля 2023 г., 15:09:15

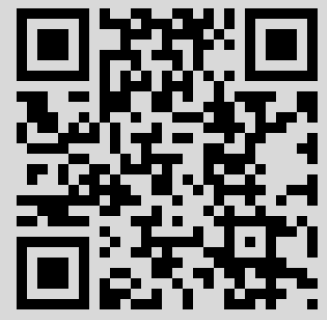




\title{
ГОМОТОПИЧЕСКИЕ ТЕОРИИ АЛГЕБР НАД ОПЕРАДАМИ
}

\author{
В. А. Смирнов
}

В работе определяются гомотопические теории алгебр над операдами и, в частности, над операдами " $n$-мерных кубиков". Построены спектральные последовательности и проведены вычисления соответствуюших гомотопических групп.

Библиография: 8 названий.

Имеются две классические теории гомотопий:

1) гомотопическая теория топологических пространств, в которой вычисление гомотопических групп сфер является одной из труднейших проблем алгебраической топологии;

2) рациональная гомотопическая теория, в которой вычисление гомотопических групп сфер - довольно простая задача.

В работе [1] было показано, что рациональная гомотопическая теория эквивалентна гомотопической теории коммутативных $D G A$-алгебр. В работах [2], [3] было показано, что сингулярный цепной комплекс $C_{*}(X)$ (коцепной комплекс $C^{*}(X)$ ) топологического пространства $X$ имеет естественную структуру $E_{\infty}$-коалгебры ( $E_{\infty}$-алгебры) и гомотопическая теория топологических пространств эквивалентна гомотопической теории $E_{\infty}$-коалгебр ( $E_{\infty}$-алгебр).

Естественньй вопрос состоит в определении промежуточных гомотопических теорий между гомотопическими теориями $D G A$-алгебр и $E_{\infty}$-алгебр, а также в вычислении гомотопических групп сфер в этих теориях.

Здесь мы определим гомотопические теории алгебр над операдами и, в частности, над операдами " $n$-мерных кубиков" $E_{n}, 1 \leqslant n \leqslant \infty$ (см. [4]). Будут построены спектральные последовательности и проведены вычисления соответствующих гомотопических групш.

Напомним, что семейство $\mathscr{E}=\{\mathscr{E}(j)\}_{j \geqslant 1}$ цепных комплексов $\mathscr{E}(j)$, на которых действуют симметрические группы $\Sigma_{j}$, называется операдой, если заданы операции

$$
\gamma: \mathscr{E}(k) \otimes \mathscr{E}\left(j_{1}\right) \otimes \cdots \otimes \mathscr{E}\left(j_{k}\right) \rightarrow \mathscr{E}\left(j_{1}+\cdots+j_{k}\right)
$$

согласованные с действиями симметрических групп и удовлетворяюшие некоторьм соотношениям ассоциативности [2].

Работа выполнена при поддержке Российского фонда фундаментальных исследований, грант № 01-01-00482. 
Цепной комплекс $X$ назьвается алгеброй (соответственно коалгеброй) над операдой $\mathscr{E}$, или просто $\mathscr{E}$-алгеброй (соответственно $\mathscr{E}$-коалгеброй), если заданы операции

$$
\mu(j): \mathscr{E}(j) \otimes X^{\otimes j} \rightarrow X \quad\left(\text { соответственно } \tau(j): X \rightarrow \operatorname{Hom}\left(\mathscr{E}(j) ; X^{\otimes j}\right)\right),
$$

согласованные с действиями симметрических групп и удовлетворяющие некоторьм соотношениям ассоциативности [2].

Обозначим сумму

$$
\sum_{j} \mathscr{E}(j) \otimes_{\Sigma_{j}} X^{\otimes j}
$$

через $\underline{\mathscr{E}}(X)$. Соответствие $X \mapsto \underline{\mathscr{E}}(X)$ определяет функтор в категории цепных комплексов. Структура операды на $\mathscr{E}$ определяет естественное преобразование функторов $\gamma: \underline{\mathscr{E}} \circ \underline{\mathscr{E}} \rightarrow \underline{\mathscr{E}}$, задающее на $\underline{\mathscr{E}}$ структуру монады [3]. При этом цепной комплекс $X$ является алгеброй над операдой $\mathscr{E}$ тогда и только тогда, когда он является алгеброй над монадой $\underline{\mathscr{E}}$.

Двойственньп образом, обозначим

$$
\overline{\mathscr{E}}(X)=\prod_{j} \operatorname{Hom}_{\Sigma_{j}}\left(\mathscr{E}(j) ; X^{\otimes j}\right) .
$$

Соответствие $X \mapsto \overline{\mathscr{E}}(X)$ определяет комонаду в категории цепных комплексов. При этом цепной комплекс $X$ является коалгеброй над операдой $\mathscr{E}$ тогда и только тог да, когда он является коалгеброй над комонадой $\overline{\mathscr{E}}$.

Заметим, что операды и алгебры над операдами могут быть определены и в категории топологических пространств. В этом случае вместо тензорного произведения $\otimes$ в определении операций $\gamma$ нужно написать обычное декартово произведение $\times$ топологических пространств [3].

Рассмотрим некоторые примеры операд и алгебр (коалгебр) над операдами.

ПримеР 1. Операда $E_{0}=\left\{E_{0}(j)\right\}$, где $E_{0}(j)$ - свободный модуль с одной нуль-мерной образующей $e(j)$ и тривиальным действием симметрических груш $\Sigma_{j}$. Таким образом, $E_{0}(j) \cong R$.

Операция $\gamma: E_{0} \times E_{0} \rightarrow E_{0}$ задается формулой

$$
\gamma\left(e(k) \otimes e\left(j_{1}\right) \otimes \cdots \otimes e\left(j_{k}\right)\right)=e\left(j_{1}+\cdots+j_{k}\right) .
$$

Легко проверяется, что так определенная операция ассоциативна и согласована с действиями симметрических групш.

Алгебрами (коалгебрами) над $E_{0}$ являются просто коммутативные и ассоциативные алгебры (коалгебры).

Пример 2. Операда $A=\{A(j)\}$, где $A(j)$ - свободньй $\Sigma_{j}$-модуль с одной нуль-мерной образующей $a(j)$, т.е. $A(j) \cong R\left(\Sigma_{j}\right)$. Операция $\gamma: A \times A \rightarrow A$ задается формулой

$$
\gamma\left(a(k) \otimes a\left(j_{1}\right) \otimes \cdots \otimes a\left(j_{k}\right)\right)=a\left(j_{1}+\cdots+j_{k}\right) .
$$

Легко проверяется, что вьполняются требуемые соотношения.

Алгебрами (коалгебрами) над операдой $A$ являются просто ассоциативные алгебры (коалгебры). 
ПРИмеР 3. Для произвольного цепного комплекса $X$ определим операды $\mathscr{E}_{X}, \mathscr{E}^{X}$, положив

$$
\mathscr{E}_{X}(j)=\operatorname{Hom}\left(X^{\otimes j} ; X\right), \quad \mathscr{E}^{X}(j)=\operatorname{Hom}\left(X ; X^{\otimes j}\right)
$$

Действия симметрических групп задаются перестановками сомножителей в $X^{\otimes j}$, a структура операды определяется формулами

$$
\begin{aligned}
& \gamma_{X}\left(f \otimes g_{1} \otimes \cdots \otimes g_{k}\right)=f \circ\left(g_{1} \otimes \cdots \otimes g_{k}\right), \quad f \in \mathscr{E}_{X}(k), \quad g_{i} \in \mathscr{E}_{X}\left(j_{i}\right) ; \\
& \gamma^{X}\left(f \otimes g_{1} \otimes \cdots \otimes g_{k}\right)=\left(g_{1} \otimes \cdots \otimes g_{k}\right) \circ f, \quad f \in \mathscr{E}^{X}(k), \quad g_{i} \in \mathscr{E}^{X}\left(j_{i}\right) \text {. }
\end{aligned}
$$

Цепной комплекс $X$ является алгеброй (соответственно коалгеброй) над операдой $\mathscr{E}$ в том и только том случае, если имеет место отображение операд $\xi: \mathscr{E} \rightarrow \mathscr{E}_{X}$ (соответственно $\left.\xi: \mathscr{E} \rightarrow \mathscr{E}^{X}\right)$.

ПРимеР 4. Для $n \geqslant 0$ обозначим через $\Delta^{n}$ нормализованньй цепной комплекс стандартного $n$-мерного симплекса. Тогда $\Delta^{*}=\left\{\Delta^{n}\right\}$ является косимплициальньм объектом в категории цепных комплексов.

Обозначим реализацию косимплициального объекта $\left(\Delta^{*}\right)^{\otimes j}=\Delta^{*} \otimes \cdots \otimes \Delta^{*}$ через $E^{\Delta}(j)$, т.е.

$$
E^{\Delta}(j)=\operatorname{Hom}\left(\Delta^{*} ;\left(\Delta^{*}\right)^{\otimes j}\right),
$$

где Hom рассматривается в категории косимплициальных объектов.

Семейство $E^{\Delta}=\left\{E^{\Delta}(j)\right\}$ является операдой, для которой действия симметрических груш и структура операды определяются аналогично соответствуюшим структурам определенной вьше операды $\mathscr{E} X$, где вместо $X$ берется $\Delta^{*}$.

Заметим, что так как цепные комплексы $\Delta^{n}$ ацикличны, то также ацикличной будет и операда $E^{\Delta}$.

В работе [3] было показано, что на сингулярном цепном комплексе $C_{*}(X)$ топологического пространства $X$ имеет место естественная структура $E^{\Delta}$-коалгебры. Двойственньм образом, на коцепном комплексе $C^{*}(X)$ имеется естественная структура $E^{\Delta}$-алгебры.

ПРИмеР 5. Основными примерами операд в категории топологических пространств являются операды $n$-мерных кубиков $\mathscr{E}_{n}$, введенные Бордманом и Фогтом [5] и изученные Мэем [4]. В частности, им было показано, что всякое $n$-кратное пространство петель $\Omega^{n} X$ является алгеброй над операдой $\mathscr{E}_{n}$.

Имеют место вложения $\mathscr{E}_{n} \rightarrow \mathscr{E}_{n+1}$, прямой предел относительно которых обозначается $\mathscr{E}_{\infty}$. Операда $\mathscr{E}_{\infty}$ является ацикличной операдой со свободным действием симметрических групп.

Всякая ацикличная операда со свободным действием симметрических групп назьвается $E_{\infty}$-операдой. Всякая алгебра (коалгебра) над $E_{\infty}$-операдой называется $E_{\infty}$-aлгеброй $\left(E_{\infty}-\right.$ коалгеброй $)$.

ПРимеР 6. Легко видеть, что если $\mathscr{E}=\{\mathscr{E}(j)\}$ - операда в категории топологических пространств, то семейство цепных комплексов $C_{*}(\mathscr{E})=\left\{C_{*}(\mathscr{E}(j))\right\}$ будет операдой в категории цепных комплексов. Если $\mathscr{E}$ является $E_{\infty}$-операдой, то $C_{*}(\mathscr{E})$ будет $E_{\infty \text {-операдой. }}$ 
Покажем, что сингулярный цепной комплекс $C_{*}(X)$ (соответственно сингулярный коцепной комплекс $\left.C^{*}(X)\right)$ является $E_{\infty}$-коалгеброй (соответственно $E_{\infty}$-алгеброй).

Пусть $E-E_{\infty}$-операда. Рассмотрим операду $E^{\Delta} \otimes E$. Она является $E_{\infty}$-операдой, и имеет место проекция операд $p: E^{\Delta} \otimes E \rightarrow E^{\Delta}$. Тогда композиция

$$
E^{\Delta} \otimes E \stackrel{p}{\rightarrow} E^{\Delta} \stackrel{\xi}{\rightarrow} \mathscr{E}^{C_{*}(X)} \quad\left(\text { соответственно } E^{\Delta} \otimes E \stackrel{p}{\rightarrow} E^{\Delta} \stackrel{\xi}{\rightarrow} \mathscr{E}_{C^{*}(X)}\right)
$$

будет задавать на $C_{*}(X)$ (соответственно $\left.C^{*}(X)\right)$ структуру $E^{\Delta} \otimes E$-коалгебры (соответственно $E^{\Delta} \otimes E$-алгебры).

Обозначим операду $E^{\Delta} \otimes C_{*}\left(\mathscr{E}_{n}\right)$ просто $E_{n}$, и будем назьвать ее также операдой $n$-мерных кубиков. Тогда $C_{*}(X)$ можно считать $E_{n}$-коалгеброй. Двойственньг образом $C^{*}(X)$ можно считать $E_{n}$-алгеброй.

Нам потребуется следующее общее свойство алгебр (коалгебр) над операдами.

Теорема 1. Если $X_{*}=\left\{X_{n}\right\}$ - симплиииальный обгект в категории алгебр над операдой $\mathscr{E}$, то его реализачия $\left|X_{*}\right|$ также будет $\mathscr{E}$-алгеброй. Двойственным образом, если $X^{*}=\left\{X^{n}\right\}-$ косимпличиальный обгект в категории коалгебр над операдой $\mathscr{E}$, то его реализачия $\left|X^{*}\right|$ также будет $\mathscr{E}$-коалгеброй.

ДокАЗАТЕЛЬСтво. Пусть $X_{*}=\left\{X_{n}\right\}$ - симплищиальный объект в категории $\mathscr{E}$-алгебр, $\mu_{n}: \mathscr{E}\left(X_{n}\right) \rightarrow X_{n}$ - структура $\mathscr{E}$-алгебрына $X_{n}$. Отображения Эйленберга-Зильбера

$$
\psi:\left|X_{*}\right| \otimes \cdots \otimes\left|X_{*}\right| \rightarrow\left|X_{*} \otimes \cdots \otimes X_{*}\right|
$$

перестановочны с действиями симметрических груп и, следовательно, индуцируют отображения

$$
\psi: \mathscr{E}(j) \otimes_{\Sigma_{j}}\left|X_{*}\right|^{\otimes j} \rightarrow\left|\mathscr{E}(j) \otimes_{\Sigma_{j}} X_{*}^{\otimes j}\right| .
$$

Эти отображения определяют отображение $\psi: \mathscr{E}\left(\left|X_{*}\right|\right) \rightarrow\left|\mathscr{E}\left(X_{*}\right)\right|$, и искомое отображение $\mathscr{E}\left(\left|X_{*}\right|\right) \rightarrow\left|X_{*}\right|$ задается как композиция

$$
\mathscr{E}\left(\left|X_{*}\right|\right) \stackrel{\psi}{\longrightarrow}\left|\mathscr{E}\left(X_{*}\right)\right| \stackrel{\mu_{*}}{\longrightarrow}\left|X_{*}\right| .
$$

СлЕДСТВИЕ. Реализация $B(\mathscr{E}, \mathscr{E}, X)$ симплициальной резольвентьы

$$
B_{*}(\mathscr{E}, \mathscr{E}, X): \mathscr{E}(X) \leftarrow \mathscr{E}^{2}(X) \leftarrow \cdots \leftarrow \mathscr{E}^{n}(X) \leftarrow \cdots
$$

над $\mathscr{E}$-алгеброй $X$ является $\mathscr{E}$-алгеброй. Причем аугментация $\eta: B(\mathscr{E}, \mathscr{E}, X) \rightarrow X$ является цепной әквивалентностью. Двойственным образом, реализация $F(\mathscr{E}$, $\mathscr{E}, X)$ косимпличиальной резольвенты

$$
F^{*}(\mathscr{E}, \mathscr{E}, X): \overline{\mathscr{E}}(X) \rightarrow \overline{\mathscr{E}}^{2}(X) \rightarrow \cdots \rightarrow \overline{\mathscr{E}}^{n}(X) \rightarrow \cdots
$$

над E⿱-коалгеброй $X$ является $\mathscr{E}$-коалгеброй. Причем аугментация $\xi: X \rightarrow F(\mathscr{E}$, $\mathscr{E}, X)$ является иепной эквивалентностью.

Перейдем теперь к теориям гомотопий. Пусть $\mathscr{E}$ - операда, для которой задано отображение операд $\mathscr{E} \rightarrow \mathscr{E}^{\Delta}$. Это означает, что цепные комплексы $\Delta^{n}$ обладают структурами $\mathscr{E}$-коалгебр, согласованньми с операторами кограней и ковырождения. Это требование довольно естественно для гомотопических теорий. В частности, оно позволяет определить в них гомотопические групшы. 
Обозначим $\mathscr{A}_{\mathscr{E}}$ категорию, объектами которой являются $\mathscr{E}$-алгебры, а морфизмамиотображения $\mathscr{E}$-алгебр.

Категория $\mathscr{A}_{\mathscr{E}}$ является замкнутой модельной категорией [6] в которой расслоения сюръективные отображения $p: X \rightarrow Y$; слабые эквивалентности - отображения, индуцирующие изоморфизм гомологий; корасслоения - отображения $i: A \rightarrow B$, удовлетворяющие свойству левого поднятия относительно тривиальных расслоений. Это означает, что для любой коммутативной диаграммы

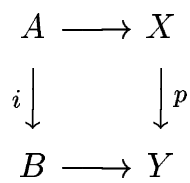

существует диагональное отображение $f: B \rightarrow X$, сохраняющее коммутативность.

Двойственньм образом, обозначим через $\mathscr{K}_{\mathscr{E}}$ категорию, объектами которой являются $\mathscr{E}$-коалгебры, а морфизмами - отображения $\mathscr{E}$-коалгебр.

Категория $\mathscr{K}_{\mathscr{E}}$ является замкнутой модельной категорией, в которой корасслоения инъективные отображения $i: A \rightarrow B$; слабые эквивалентности - отображения, индуцирующие изоморфизм гомологий; расслоения - отображения $p: X \rightarrow Y$, удовлетворяющие свойству правого поднятия относительно тривиальных корасслоений. Это означает, что для любой рассмотренной выше коммутативной диаграммы существует диагональное отображение $f: B \rightarrow X$, сохраняющее коммутативность.

Теорема 2. Для любого тривиального расслоения $p: X \rightarrow Y$ в категории $\mathscr{A}_{\mathscr{E}}$ сущ,ствует отображсение $\mathscr{E}$-алгебр $\tilde{q}: B(\mathscr{E}, \mathscr{E}, Y) \rightarrow X$ такое, что

$$
p \circ \tilde{q}=\eta: B(\mathscr{E}, \mathscr{E}, Y) \rightarrow Y .
$$

ДокАЗАТЕЛЬСтво. Пусть $p: X \rightarrow Y$ - тривиальное расслоение. Это означает, что $p$ сюрьективно и индуцирует изоморфизм гомологий. Следовательно, существует цепное отображение $q: Y \rightarrow X$ и цепная гомотопия $h: X \rightarrow X$ такие, что

$$
p \circ q=\mathrm{Id}, \quad d(h)=q \circ p-\mathrm{Id}, \quad p \circ h=0, \quad h \circ q=0, \quad h \circ h=0 .
$$

Построим отображение $\mathscr{E}$-алгебр $\tilde{q}: B(\mathscr{E}, \mathscr{E}, Y) \rightarrow X$.

Легко видеть, что задание такого отображения эквивалентно заданию семейства отображений $\mathscr{E}$-алгебр $q^{n}: \mathscr{E}^{n+1}(Y) \rightarrow \operatorname{Hom}\left(\Delta^{n} ; X\right)$, для которого коммутативны диаграммы

$$
\begin{array}{crr}
\mathscr{E}^{n}(Y) & \stackrel{q^{n-1}}{\longrightarrow} & \operatorname{Hom}\left(\Delta^{n-1} ; X\right) \\
s_{i} \downarrow \uparrow d_{i} & s_{i} \downarrow \uparrow d_{i} \\
\mathscr{E}^{n+1}(Y) \stackrel{q^{n}}{\longrightarrow} & \operatorname{Hom}\left(\Delta^{n} ; X\right)
\end{array}
$$

Так как задание отображения $\mathscr{E}$-алгебр $q^{n}: \mathscr{E}^{n+1}(Y) \rightarrow \operatorname{Hom}\left(\Delta^{n} ; X\right)$ эквивалентно заданию цепного отображения $\bar{q}^{n}: \mathscr{E}^{n}(Y) \rightarrow \operatorname{Hom}\left(\Delta^{n} ; X\right)$, то задание отображения $\mathscr{E}$-алгебр $\tilde{q}: B(\mathscr{E}, \mathscr{E}, Y) \rightarrow X$ эквивалентно заданию семейства цепных отображений $\bar{q}^{n}: \mathscr{E}^{n}(A) \rightarrow \operatorname{Hom}\left(\Delta^{n} ; X\right)$, для которых соответствуюшие отображения $\mathscr{E}$-алгебр $f^{n}$ дают приведенные вьше коммутативные диаграммы.

Положим $\bar{q}^{0}=q: Y \rightarrow X$ и $\bar{q}^{n}=h \circ \mu \circ \mathscr{E}(h) \circ \mathscr{E}(\mu) \circ \cdots \circ \mathscr{E}^{n-1}(\mu) \circ \mathscr{E}^{n}(q)$. Прямые вычисления показывают, что выполняются требуемые соотношения. 


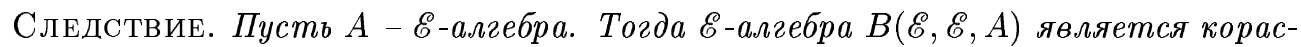
слоенным обгектом в категории $\mathscr{A}_{\mathscr{E}}$.

Действительно, пусть $p: X \rightarrow Y$ - тривиальное расслоение, $f: B(\mathscr{E}, \mathscr{E}, A) \rightarrow Y$ - отображение $\mathscr{E}$-алгебр. Построим отображение $\mathscr{E}$-алгебр $\tilde{f}: B(\mathscr{E}, \mathscr{E}, A) \rightarrow X$, для которого $p \circ \widetilde{f}=f$.

В силу доказанной теоремы мы имеем отображение $\mathscr{E}$-алгебр $\tilde{q}: B(\mathscr{E}, \mathscr{E}, Y) \rightarrow X$. Так как $\eta: B(\mathscr{E}, \mathscr{E}, A) \rightarrow A$ - тривиальное расслоение, то существует отображение $\mathscr{E}$-алгебр $\psi: B(\mathscr{E}, \mathscr{E}, A) \rightarrow B(\mathscr{E}, \mathscr{E}, B(\mathscr{E}, \mathscr{E}, A))$. Определим $\widetilde{q}$ как композицию

$$
B(\mathscr{E}, \mathscr{E}, A) \stackrel{\psi}{\longrightarrow} B(\mathscr{E}, \mathscr{E}, B(\mathscr{E}, \mathscr{E}, A)) \stackrel{B(\mathscr{E}, \mathscr{E}, f)}{\longrightarrow} B(\mathscr{E}, \mathscr{E}, Y) \stackrel{\tilde{q}}{\rightarrow} X
$$

Двойственньгм образом, имеем

Теорема $2^{\prime}$. Для любого тривиального корасслоения $i: A \rightarrow B$ в категории $\mathscr{K}_{\mathscr{E}}$ существует отображсение $\mathscr{E}$-коалгебр $\tilde{j}: B \rightarrow F(\mathscr{E}, \mathscr{E}, A)$, для которого

$$
\tilde{j} \circ i=\xi: A \rightarrow F(\mathscr{E}, \mathscr{E}, A) \text {. }
$$

СледСТВИЕ. Пусть $X-\mathscr{E}-к о а л г е б р а . ~ Т о г д а ~ \mathscr{E - к о а л г е б р а ~} F(\mathscr{E}, \mathscr{E}, X)$ является расслоенным обгектом в категории $\mathscr{K}_{\mathscr{E}}$.

Рассмотрим категорию $\widetilde{\mathscr{K}}_{\mathscr{E}}$, объектами в которой являются $\mathscr{E}$-коалгебры, а морфизмами $f: X \rightarrow Y$ - отображения $\mathscr{E}$-коалгебр $\tilde{f}: X \rightarrow F(\mathscr{E}, \mathscr{E}, Y)$.

Два морфизма $f_{0}, f_{1}: X \rightarrow Y$ категории $\widetilde{\mathscr{K}}_{\mathscr{E}}$ будем назьвать гомотопным. и и обозначать $f \simeq g$, если существует морфизм $h: \Delta^{1} \otimes X \rightarrow Y$, назьваемьй гомотопией, такой, что

$$
\left.h\right|_{0 \otimes X}=f_{0},\left.\quad h\right|_{1 \otimes X}=f_{1} .
$$

Обозначим $Н о \mathscr{K}_{\mathscr{E}}$ локализацию категории $\mathscr{K}_{\mathscr{E}}$ по отношению к слабьм эквивалентностям, т.е. морфизмам, индуцирующим изоморфизмы гомологий.

Пусть $\pi \mathscr{K}_{\mathscr{E}}$ обозначает категорию, объектами которой являются $\mathscr{E}$-коалгебры, а морфизмами гомотопические классы морфизмов категории $\widetilde{\mathscr{K}}_{\mathscr{E}}$. Общая теория гомотопий в категориях [6] дает следующий результат.

ТЕОРема 3. Имеет место әквивалентность категорий

$$
H o \mathscr{K}_{\mathscr{E}} \cong \pi \mathscr{K}_{\mathscr{E}} .
$$

Двойственньгм образом, для $\mathscr{E}$-алгебр имеем

ТЕОремА $3^{\prime}$. Имеет место әквивалентность категорий

$$
H o \mathscr{A}_{\mathscr{E}} \cong \pi \mathscr{A}_{\mathscr{E}}
$$

Рассмотрим теперь проблему вычисления гомотопических груп $\mathscr{E}$-коалгебр. Так как цепные комплексы $\Delta^{n}$ стандартных $n$-мерных симплексов являются $\mathscr{E}$-коалгебрами, то цепные комплексы $S^{n} n$-мерных сфер также будут $\mathscr{E}$-коалгебрами.

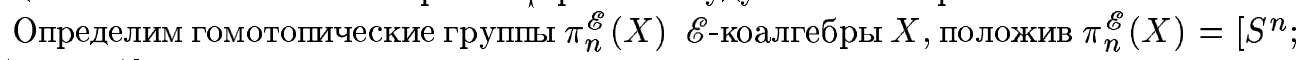
$F(\mathscr{E}, \mathscr{E}, X)]$ - множество гомотопических классов отображений $\mathscr{E}$-коалгебр $f: S^{n} \rightarrow$ $F(\mathscr{E}, \mathscr{E}, X)$. 
ТеоремА 4. Для произвольной Ё-коалгебры X имеет место спектральная последовательность гомотопических групп $\pi_{*}^{\mathscr{E}}(X)$, член $E^{1}$ которой изоморфен кобар конструкиии $F\left(\mathscr{E}_{*}, X_{*}\right)$, где $\mathscr{E}_{*}, X_{*}$ обозначают гомологии $\mathscr{E}$ u $X$ соответственно.

ДокАЗАТЕЛЬСТво. Рассмотрим фильтрацию

$$
F(\mathscr{E}, \mathscr{E}, X) \supset F^{1}(\mathscr{E}, \mathscr{E}, X) \supset \cdots \supset F^{m}(\mathscr{E}, \mathscr{E}, X) \supset \cdots
$$

где $F^{m}(\mathscr{E}, \mathscr{E}, X): \overline{\mathscr{E}}^{m}(X) \rightarrow \overline{\mathscr{E}}^{m+1}(X) \rightarrow \cdots$.

Она индуцирует спектральную последовательность. Точные последовательности

$$
0 \rightarrow F^{m+1}(\mathscr{E}, \mathscr{E}, X) \rightarrow F^{m}(\mathscr{E}, \mathscr{E}, X) \rightarrow \overline{\mathscr{E}}^{m+1}(X) \rightarrow 0
$$

индуцируют изоморфизмы

$$
E_{n, m}^{1}=\left[S^{n}, \overline{\mathscr{E}}^{m+1}(X)\right] \cong H_{n}\left(\overline{\mathscr{E}}^{m}(X)\right)
$$

и, следовательно, изоморфизм $E^{1} \cong F\left(\mathscr{E}_{*}, X_{*}\right)$.

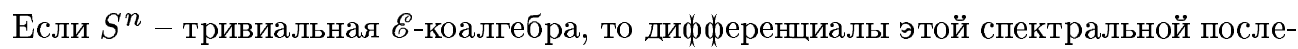
довательности определяются дифференциалами в кобар конструкции $F(\mathscr{E}, X)$ и, таким образом, мы имеем следующий результат.

ТЕОремА 5. Если $S^{n}$ - тривиальная $\mathscr{E}-к о а л г е б р а, ~ т о ~ д л я ~ п р о и з в о л ь н о и ̆ ~ \mathscr{- к о - ~}$ алгебры $Х$ имеет место изоморфизм

$$
\pi_{n}^{\mathscr{E}}(X) \cong H_{n}(F(\mathscr{E}, X)) .
$$

Пусть теперь $X$ - топологическое пространство, $E_{n}$ - операда $n$-мерньх кубиков. Заметим, что если $m \geqslant n$, то $S^{m}$ имеет тривиальную структуру $E_{n}$-коалгебры, откуда вытекает следующая

ТЕОрема 6. Если $X$ - топологическое пространство и $m \geqslant n$, то имеет место изоморфизм

$$
\pi_{m}^{E_{n}}(X) \cong H_{m}\left(F\left(E_{n}, C_{*}(X)\right)\right)
$$

Член $E^{1}$ этой спектральной последовательности выражается через гомологии операды $E_{n}$ и, следовательно, через алгебру Дайера-Лашофа [7], [8].

ТЕорема 7. Если $X$ - топологическое пространство, то член $E^{1}$ спектральной последовательности гомотопических групп $\pi_{*}^{E_{n}}(X)$ изоморфен модулю $S^{n} T_{s} R_{n-1} L_{n-1} S^{-n} H_{*}(X)$, әде $T_{s}$ - свободная коммутативная алгебра, $R_{n-1}$ подмодуль алгебры Дайера-Лашофа, порохсденный допустимыми последовательностями избьточности меньше $n, L_{n-1}-$ свободная $(n-1)$-алгебра Лu.

Если $X-n$-связное топологическое пространство, то кобар конструкция $F\left(E_{n}\right.$, $\left.C_{*}(X)\right)$ цепно эквивалентна $n$-кратной надстройке над цепным комплексом итерированного пространства петель $\Omega^{n} X[8]$. Следовательно, имеет место 
ТЕоРема 8. Если $X$ - n-связное топологическое пространство, то имеет место изоморфизм

$$
\pi_{*}^{E_{n}}(X) \cong S^{n} H_{*}\left(\Omega^{n} X\right) .
$$

Эта теорема обобщает результат Квиллена [1], [6] о том, что рациональные гомотопические группы односвязного топологического пространства выражаются через гомологии его пространства петель. Она устанавливает, до какого $m$ остается непосредственная связь между гомотопическими группами (над операдой $\left.E_{m}\right) n$-связного пространства $X$ и гомологиями $m$-кратного пространства петель над $X$.

Предложенньй метод позволяет заменить труднейшую задачу о вычислении гомотопических групा сфер над операдой $E_{\infty}$ на задачу о вычислении гомотопических групा сфер на операдами $E_{n}$.

В частности, из доказанной теоремы следует, что если $m \leqslant n$, то гомотопические группы (над операдой $E_{m}$ ) сферы $S^{n}$ изоморфны $m$-кратной надстройке над гомологиями $m$-кратного пространства петель над $S^{n}$. Интересно, на наш взгляд, было бы вычислить гомотопические группы сферы $S^{n}$ над операдами $E_{n}, E_{n+1}$ и т.д.

\section{СПИСОК ЦИТИРОВАННОЙ ЛИТЕРАТУРЫ}

[1] Quillen D. Rational homotopy theory // Ann. of Math. 1969. V. 90. № 2. P. 205-295.

[2] Смирнов В.А. О коцепном комплексе топологического пространства // Матем. сб. 1981. T. 115 . C. $146-158$.

[3] Смирнов В.А. Гомотопическая теория коалгебр // Изв. АН СССР. Сер. матем. 1985. T. 49. C. $1302-1321$.

[4] May J.P. The Geometry of Iterated Loop Spaces. Lecture Notes in Math. V. 271. New York: Springer, 1972.

[5] Boardman J. M., Vogt R. M. Homotopy Invariant Algebraic Structures on Topological Spaces. Lecture Notes in Math. V. 347. New York: Springer, 1973.

[6] Quillen D. Homotopical Algebra. Lecture Notes in Math. V. 43. New York: Springer, 1967.

[7] Dyer E., Lashof R. Homology of iterated loop spaces // Amer. J. Math. 1962. V. 84. P. $35-88$.

[8] Smirnov V. A. The homology of iterated loop spaces // Forum Mathematicum. 2002. V. 14. P. $345-381$. 